Z Herz- Thorax- Gefäßchir 2021 · 35:283-290 https://doi.org/10.1007/s00398-021-00453-y Eingegangen: 1. Juli 2021

Angenommen: 1. Juli 2021

Online publiziert: 15 . September 2021

( $)$ Springer Medizin Verlag GmbH, ein Teil von Springer Nature 2021

\section{Patiententransport und Netzwerke beim Einsatz des Extracorporeal Life Support}

\author{
F. Born ${ }^{1} \cdot$ C. Müller' $\cdot$ M. Hanuna ${ }^{1} \cdot$ U. Boeken ${ }^{2} \cdot$ C. Hagl' \\ 'Herzchirurgische Klinik und Poliklinik, Klinikum der LMU, München, Deutschland \\ ${ }^{2}$ Klinik für Herzchirurgie, Universitätsklinik Düsseldorf, Düsseldorf, Deutschland
}

\title{
Zusammenfassung
}

Neue Technologien und die kontinuierliche Weiterentwicklung extrakorporaler Unterstützungssysteme haben das Anwendungsspektrum des Extracorporeal Life Support (ECLS) in den letzten Jahren erweitert. Neben dem Einsatz im kardiogenen Schock oder unter Reanimation nehmen die Anfragen zur Übernahme instabiler Patienten aus peripheren Krankenhäusern zu. Durch organisatorische Herausforderungen wie die Etablierung von Netzwerken und ein strukturiertes Teamtraining aller Beteiligten ergibt sich eine rasche Verfügbarkeit des ECLS-Teams, um schnell beim zu versorgenden Patienten einzutreffen.

\section{Schlüsselwörter}

Kardiopulmonale Reanimation · Organisation · Equipment · Komplikationen · Training

Neue Technologien und die kontinuierliche Weiterentwicklung extrakorporaler Unterstützungssysteme haben das Anwendungsspektrum des Extracorporeal Life Support (ECLS) in den letzten Jahren erweitert. Neben dem Einsatz im kardiogenen Schock oder unter Reanimation nehmen die Anfragen zur Übernahme instabiler Patienten aus peripheren Krankenhäusern zu. Damit ergeben sich neue Herausforderungen an die Technologien, aber auch an das ECLS-Team. AuBer der generellen Bereitstellung bedarf es einer Infrastruktur, die den Transport und die Sicherheit der Patienten jederzeit gewährleistet. Diese Aspekte sind unter Berücksichtigung wirtschaftlicher Betrachtungen, aber auch der gesellschaftlichen Verantwortung eine gemeinsame Herkulesaufgabe.

Die ECLS-Therapie hat sich als Standardverfahren bereits bewährt und wurde 2015 in die Leitlinien zur Reanimation des European Resuscitation Council (ERC) als Empfehlung aufgenommen [1]. Im Jahr 2019 hat der Gemeinsame Bundesausschuss (G$B A) u$. a. folgende Zentrumsregelungen gemäß § 136c Abs. 5 SGB V beschlossen [2]:
24/7-Vorhaltung eines Abholdienstes für Patientinnen und Patienten mit venoarterieller extrakorporaler Membranoxygenierung (vaECMO) im kardiogenen Schock, bestehend aus mindestens einer Fachärztin oder einem Facharzt für Herzchirurgie oder Kardiologie oder Anästhesiologie sowie einer Kardiotechnikerin oder einem Kardiotechniker mit jeweils Erfahrung in der Implantation von va-ECMO-Systemen.

Die Gründung von Kompetenzzentren sowie die Frage der notwendigen personellen und apparativen Ausstattung wurden entsprechend in einem Positionspapier mit einer Vielzahl von Fachgesellschaften 2019 publiziert [3]. Aus herzchirurgischer/kardiotechnischer Sicht erscheint die Betreuung dieser häufig multimorbiden Patienten in kardiochirurgischen Zentren von Vorteil, da eine Bündelung operationstechnischer, apparativer und intensivmedizinischer Expertise vorliegt. In Anbetracht dessen wurden eine S3Leitlinie, koordiniert durch die Arbeitsgemeinschaft der Wissenschaftlichen Medizinischen Fachgesellschaften e.V. (AWMF [4]), erstellt. Dennoch bedarf es aufgrund der limitierten Intensivbettenressourcen 


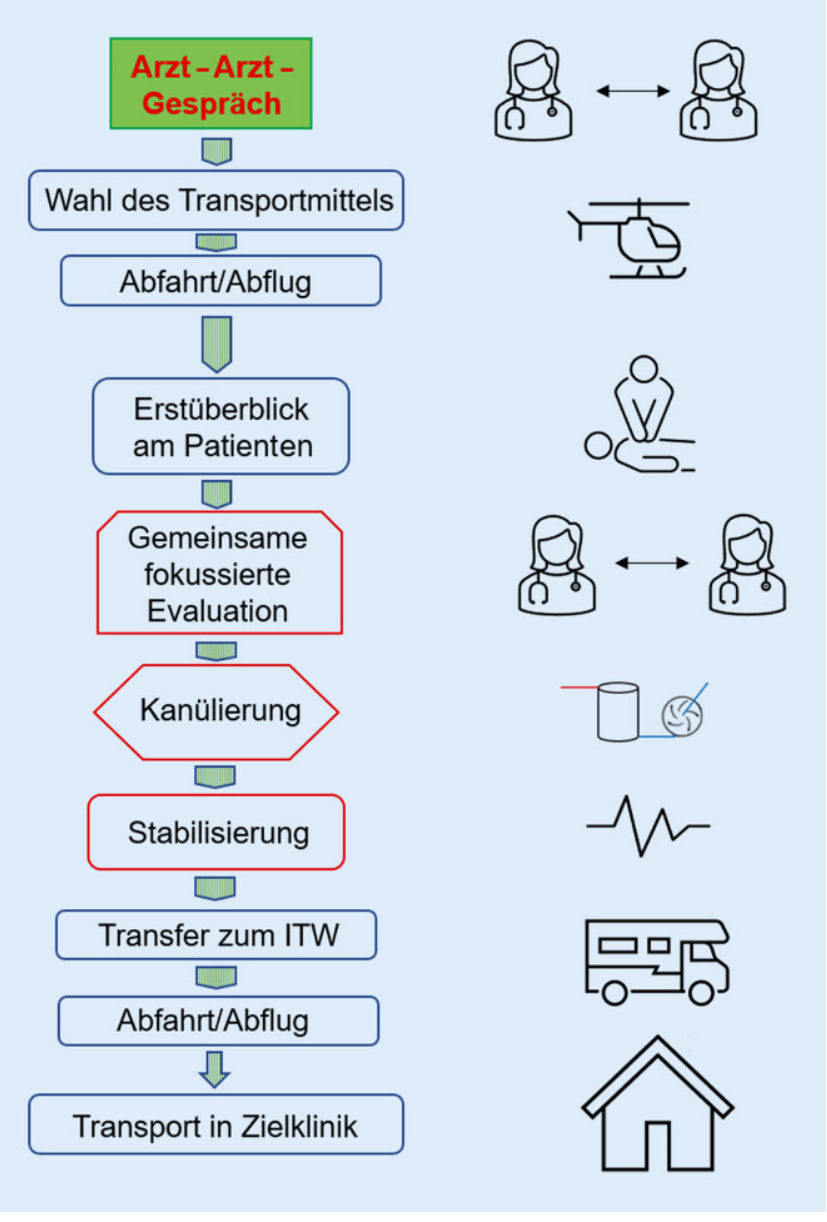

Patienten auf der Intensivstation eine große Herausforderung für die Mitarbeiter dar. Dies gilt umso mehr in kleineren Zentren für extrakorporale Membranoxygenierung (ECMO)/ECLS mit geringer Expertise. Die multidisziplinäre Einarbeitung von Ärzten und Pflegekräften auf den Intensivstationen sowie die Ausbildung und Schulung durch ECLS-Mentoren hat eine besondere Bedeutung.

Alle relevanten Berufsgruppen (Intensivmediziner, Intensivpflege, Kardiologen, Rettungsdienst, Herzkatheter[HK]-Labor etc.) müssen entsprechend in das ECLSVerfahren eingewiesen (beachte: gemäß $\$ 10$ der Medizinprodukte-Betreiberverordnung [MPBetreibV] ist das Verfahren einweisungspflichtig) und trainiert werden.

\section{Datenerhebung}

Mithilfe von Checklisten für die Gerätschaften und ihrer genauer Kontrolle können technische Fehler im Vorfeld vermieden werden. Vor Ort werden im ECLS-Implantationsprotokoll relevante Daten wie z.B. Reanimationszeit, beobachteter Stillstand, Lactat-, pH-Wert etc. dokumentiert und später ausgewertet. Während der Intensivtherapie dokumentieren KardiotechnikerInnen bei der täglichen ECLS-Visite alle wichtigen Parameter wie Blut-, Gasfluss, inspiratorische Sauerstofffraktion $\left(\mathrm{F}_{1} \mathrm{O}_{2}\right)$, Drücke des Systems (Sauerstoff- $\left[\mathrm{pO}_{2}\right]$ und Kohlendioxidpartialdruck $\left[\mathrm{pCO}_{2}\right]$ ) sowie Laborparameter (D-Dimere, Lactat, $\mathrm{pH}$ Wert, Laktatdehydrogenase [LDH] etc.) im ECLS-Protokoll. Während der WeaningPhase werden entsprechende Parameter (Katecholaminbedarf, „mean arterial pressure" [MAP], zentraler Venendruck [ZVD], Ultraschall etc.) im Weaning-Protokoll erfasst.

\section{Alarmierung - Ressourcen der Intensivstation}

An einem ECLS-Zentrum ist das Einrichten einer zentralen Telefonnummer, die 24/7 zu erreichen ist, notwendig. Diese Informationszentrale koordiniert vorhandene Kapazitäten auf den Intensivstationen und eruiert den aktuellen Personalbedarf für die ECLS-Therapie. Das ECLS-Team ist 


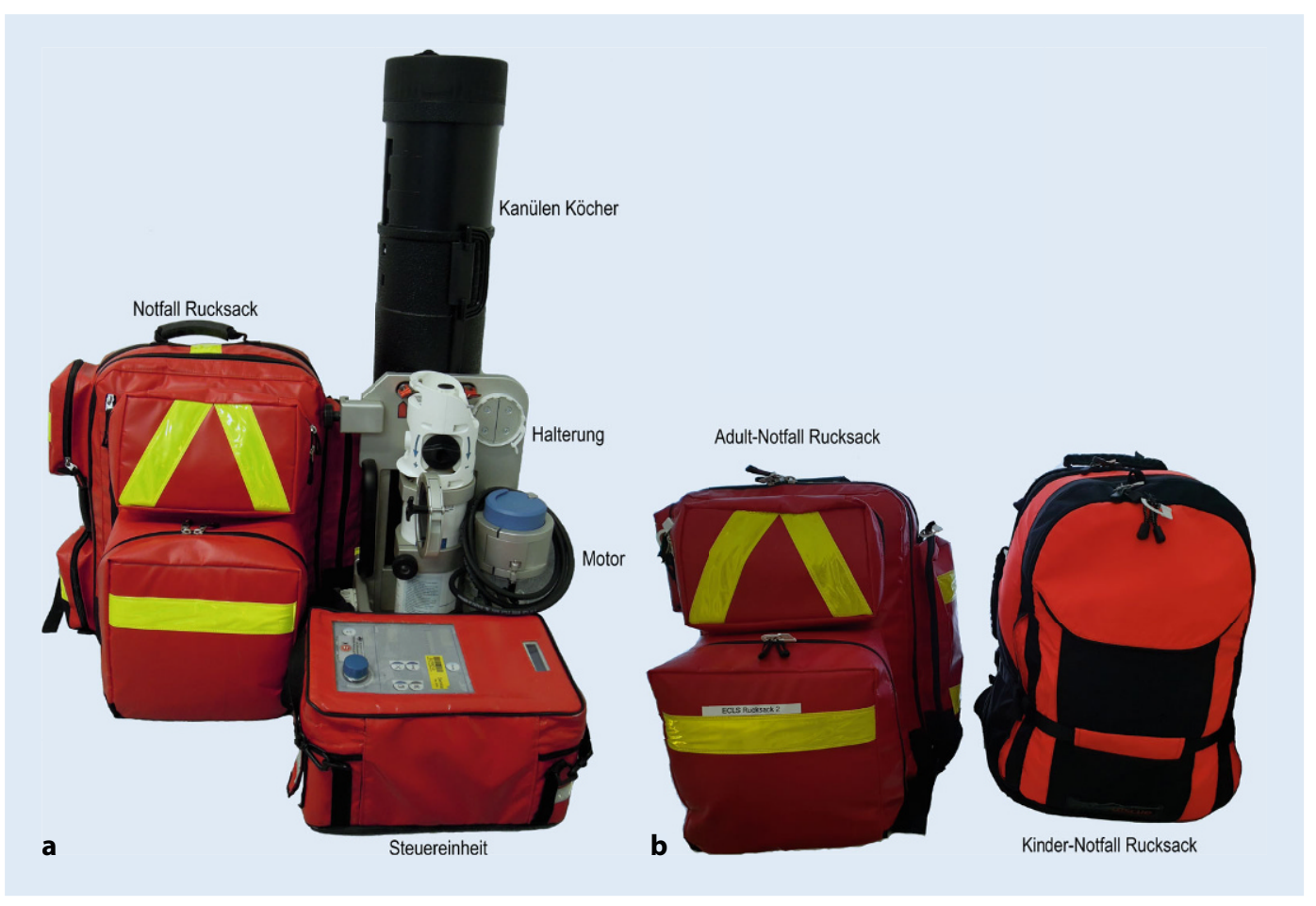

Abb. $2 \triangleleft$ Transport-Equipment. (Mit freundl. Genehmigung $\odot$ F. Born, München. Alle Rechte vorbehalten.) fester Bestandteil dieser Informationskette

(• Abb. 1).

Nach checklistenbasierter Evaluation (Arzt-zu-Arzt-Gespräch) und Indikation für eine ECLS-Implantation startet das Team die Organisation des Transports. Die Wahl des Transportmittels ist von der Entfernung der anfordernden Stelle abhängig. Primär ist es wichtig, schnell zum Patienten zu gelangen; dafür steht an der LMU ein entsprechendes Fahrzeug zur Verfügung.

\section{Technische Voraussetzungen - zum Transport geeignete Systeme}

\section{Transport-Equipment}

Für den Transport stehen 2 verplombte Rucksäcke (für Kinder und Erwachsene) zur Verfügung. In einem schwarzen Köcher befinden sich alle Kanülen in unterschiedlichen Größen und in 2-facher Menge (• Abb. 2). Zusätzlich zur $\mathrm{O}_{2}$-Flasche befindet sich ein Ambu-Beutel im Equipment. Falls die $\mathrm{O}_{2}$-Flasche leer sein sollte, kann zumindest mit Raumluft ventiliert und die Hyperkapnie reduziert werden.

Die in - Abb. 3 dargestellten Systeme sind für Patiententransporte geeignet und zugelassen. Je nach Hersteller müssen die Transportsysteme zusätzlich mithilfe von
Adapterplatten im Transportmittel fixiert werden.

\section{Kanülierung}

Das Kanülieren kann schneller und vereinfacht werden, wenn die anfordernde Klinik den Patienten bereits mit SeldingerDrähten versorgt. Durch Ultraschallvisualisierung der Gefäße hat sich die perkutane Kanülierung bei ECLS-Implantationen weiter durchgesetzt.

\section{॥) Limitierender Faktor der Generierung von Blutflussraten $>5 \mathrm{l} / \mathrm{min}$ sind die Outflow-Kanülen}

Die periphere arterielle Kanülierung kann entweder direkt perkutan in SeldingerTechnik oder offen chirurgisch durch Aufnähen einer Gefäßprothese auf die A. femoralis vorgenommen werden. Die zuletzt genannte Technik wird insbesondere bei kleinen Gefäßdurchmessern oder bei Patienten mit einer peripher-arteriellen Verschlusskrankheit eingesetzt. Bei kardiopulmonaler Reanimation wird der Anschluss an das ECLS-System primär perkutan mithilfe der Seldinger-Draht-Technik angestrebt, was unter diesen Bedingungen erschwert sein kann.
Bisdas et al. [6] berichten über Gefäßkomplikationen nach peripherer Punktion der Leistengefäße und Implantation der Kanülen (Gefäßdissektionen, falsche Aneurysmen, Hämatome, Beinischämien). Die Gefäßkomplikationen waren jedoch nicht mit einem schlechteren "Outcome" assoziiert. Wie die Praxis oft zeigt, sind die Outflow-Kanülen der limitierende Faktor bei der Generierung von Blutflussraten von mehr als $5 \mathrm{I} / \mathrm{min}$. Berdajs et al. [7] konnten im Tierexperiment die Vorteile der Smart ${ }^{\circledR}$ Kanüle zeigen; der negative „outflow pressure" ist deutlich reduziert.

\section{Ergebnisse}

Die Anwendung der ECLS-Therapie bei Patienten z.B. mit therapierefraktärem Herz-Kreislauf-Versagen/-Stillstand und/ oder unter Reanimation ist mit einem Anstieg der Überlebensrate assoziiert [8-11] und mit einer Erhöhung des günstigen neurologischen Ergebnisses verbunden [12]. Besonders durch die Verbesserung des neurologischen Outcome erscheint die Etablierung eines ECLS-Programms zur Verbesserung der Reanimationsversorgung vielversprechend. 
a

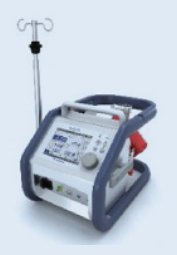

b
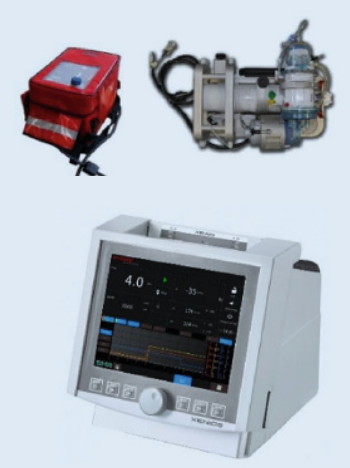

c

d

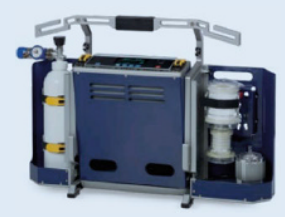

Fluss: $\max .7 \mathrm{I} / \mathrm{min}$

Drehzahl: bis $5.000 \mathrm{U} / \mathrm{min}$

Akkubetrieb: bis 90 min (belastungsabhängig)

Einsatzdauer: 30 Tage

Gewicht: ca. 20 kg (inklusive Halter)

Fluss: $\max .8 \mathrm{l} / \mathrm{min}$

Drehzahl: bis $3.500 \mathrm{U} / \mathrm{min}$

Akkubetrieb: bis 180 min (belastungsabhängig)

Einsatzdauer: 5 Tage

Gewicht: ca. 20 kg (inklusive Halter)

Fluss: $\max .8 \mathrm{l} / \mathrm{min}$

Drehzahl: bis $10.000 \mathrm{U} / \mathrm{min}$

Akkubetrieb: bis 90 min (belastungsabhängig)

Einsatzdauer: 29 Tage

Gewicht: ca. 15 kg (inklusive Halter)

Fluss: $\max .8 \mathrm{l} / \mathrm{min}$

Drehzahl: bis $5.500 \mathrm{U} / \mathrm{min}$

Akkubetrieb: bis 90 min (belastungsabhängig)

Einsatzdauer: 30 Tage

Gewicht: > 25 kg (inklusive Halter)
Abb. $3<$ Transportsysteme. a Cardiohelp (Fa. Maquet, Hechingen, Deutschland), b LifeBox (Fa. LivaNova, München, Deutschland), c Xenios Konsole (Fa. Fresenius, Bad Homburg, Deutschland), d CentriMAG (Fa. Abbott). $B T B$ "bridge to bridge", $B T R$ "bridge to recovery" ,HLM Herz-Lungen-Maschine, va venoarteriell, vv venovenös. (a, d Mit freundl. Genehmigung () CORMED Medizintechnik GmbH \& Co. KG. Alle Rechte vorbehalten. $\mathbf{b}$ Mit freundl. Genehmigung $\odot$ LivaNova Deutschland GmbH. Alle Rechte vorbehalten. c Mit freundl. Genehmigung (c) Xenios AG. Alle Rechte vorbehalten)

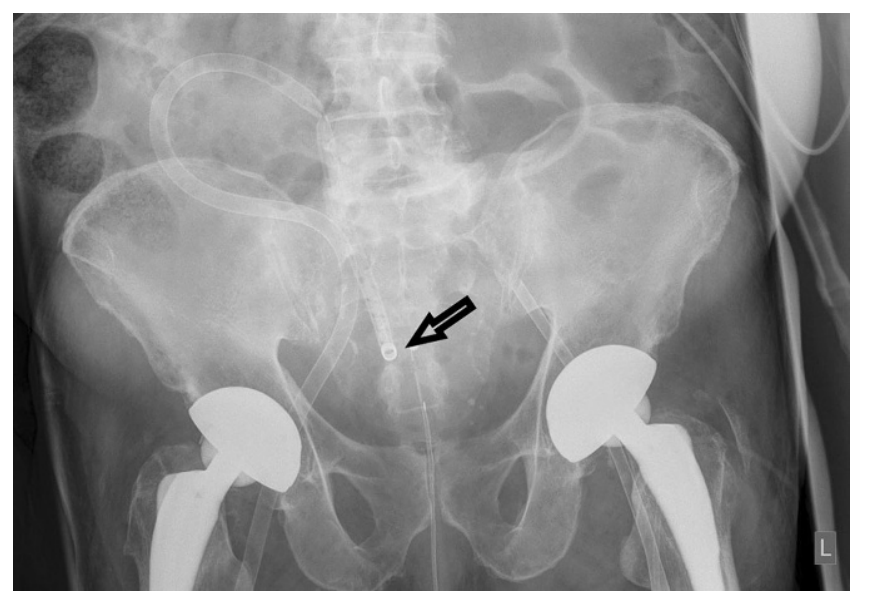

Abb. $4 \triangleleft$ Kinking der Kanüle (Pfeil) in der rechten A. ilia$\mathrm{ca}$ (Mit freundl. Genehmigung $\odot$ LMU, München. Alle Rechte vorbehalten)

\section{Mögliche Komplikationen}

Komplikationen der mechanischen Kreislaufunterstützung können sein:

- Blutung,

- Gefäßruptur,

- Beinischämie und Kompartmentsyndrom,

- Thromboembolie,

- Luftembolie,

- Kanülenfehllage, -dislokation, -Kinking (- Abb. 4),

- technische Probleme des ECLS-Systems.

\section{Transport}

Der Transport von Patienten mit extrakorporaler Zirkulation wird bereits seit vielen 


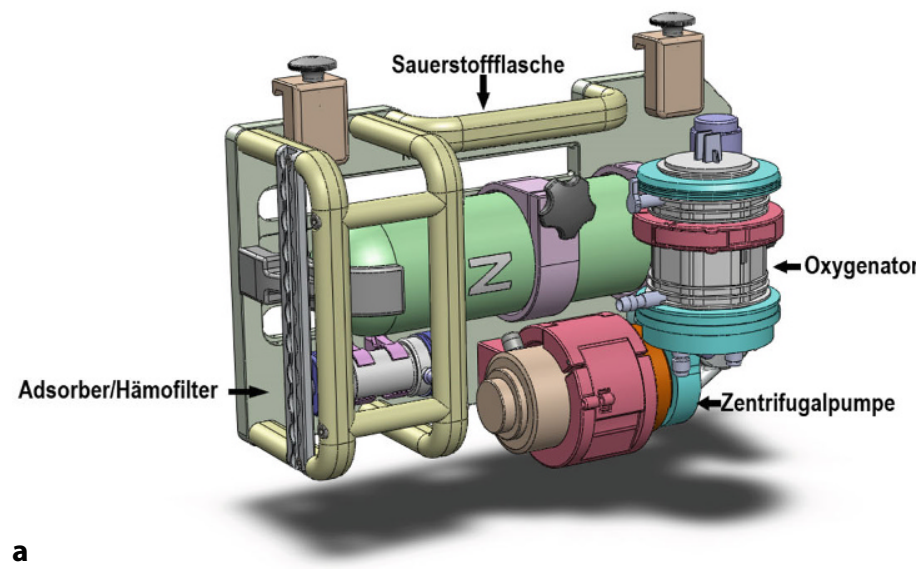

b

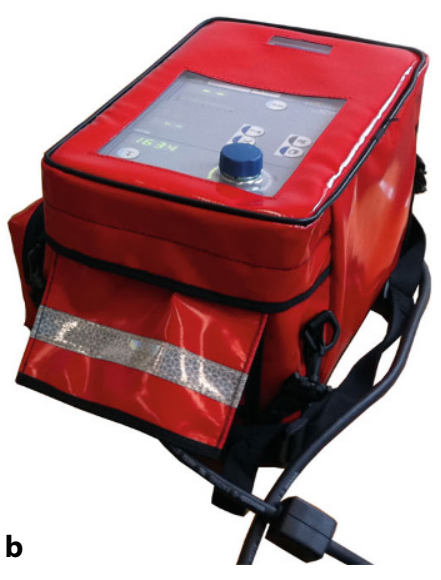

Abb. $5 \triangleleft$ Transportables Perfusionsequipment mit Hämoadsorption/ -filtration. a Halter, b LifeBox. (Mit freundl. Genehmigung $\odot$ F. Born, München. Alle Rechte vorbehalten)

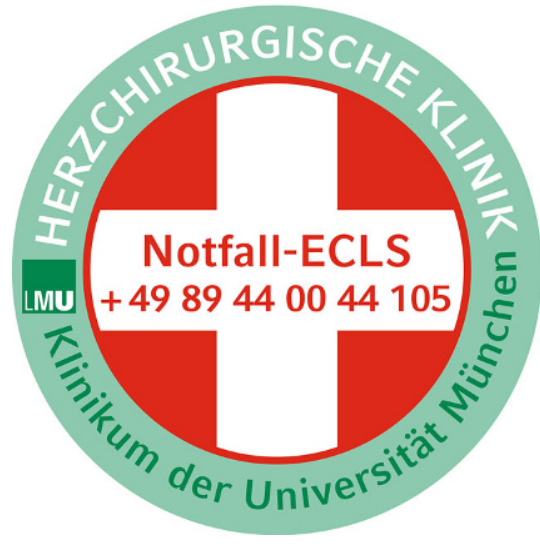

Abb. $6 \triangle$ Aufkleber Cardiac Survival Network an der Ludwig-Maximilians-Universität (LMU) München. (Mit freundl. Genehmigung $\odot$ LMU, München. Alle Rechte vorbehalten.)

Jahren global praktiziert, wie u. a. Coppola et al. [13] berichten. Zwar wurden weite Distanzen mithilfe eines Transportflugzeugs zurückgelegt, allerdings aufgrund fehlender Druckkabine in einer Flughöhe von max. $1500 \mathrm{~m}$.

Werden Intensivpatienten zwischen Kliniken verlegt, ist es meist erforderlich, die eingeleitete Intensivtherapie auch während des Transports fortzuführen. Dieser Anspruch stellt alle Beteiligten technisch und personell vor andere Herausforderungen als in der Notfallrettung.

\section{》) Die Systeme müssen klein, leicht, einfach zu bedienen sein und ausreichende Akkukapazität aufweisen}

Derzeit befinden sich wenige zugelassene Systeme auf dem Markt, die bezüglich ihres Handlings sowie ihrer Biokompatibilität und Bedienbarkeit für den mobilen ECLSEinsatz geeignet sind. Diese Transportsysteme ( $\mathrm{Abb} .3$ und 5 ) wurden speziell für den Einsatz außerhalb der herzchirurgischen Klinik entwickelt, um Patienten in Notfallsituationen zu stabilisieren und in die nächste geeignete Klinik zu transportieren. Erschwerte örtliche Gegebenheiten erfordern besondere Anforderungen an solche Transportsysteme. Sie müssen klein, leicht, einfach zu bedienen sein und über ausreichende Akkukapazität verfügen.

Finden diese Transporte in der Nacht oder bei sehr schlechten Witterungsbedingungen (Nebel) statt, können die meisten Luftrettungsorganisationen nicht fliegen, sodass bodengebunden transportiert werden muss. Die Praxis zeigt, dass z. B. das Umlagern von Patienten, ein Wechsel der Perfusoren und lokal beengte Verhältnisse (Aufzüge, über mehrere Ebenen) enorm viel Zeit und somit Batterieleistung benötigen. Gerade in ländlichen Regionen [14] sind Rettungstransportwagen (RTW) selten mit einer Netzspannung von $220 \mathrm{~V}$ an Bord ausgestattet. Die tatsächliche Transportzeit kann in manchen Fällen die Akkukapazität eines Transportsystems deutlich überschreiten.

Für den Patiententransport müssen gesetzliche Vorschriften der European Aviation Safety Agency (EASA [15]) zur Befestigung solcher ECLS-Systeme beachtet und eingehalten werden. Die Vorgaben für den Lufttransport sind deutlich höher. Ein Haltersystem muss bei Fixation z. B. in einem Helikopter die in $\bullet$ Tab. 1 aufgeführten Schwerkraftanforderungen erfüllen.

\section{Netzwerke}

Bei der patientenorientierten Versorgung in Netzwerken steht das medizinische Outcome (Qualität) im Vordergrund. Ziel ist es, benötigte Kompetenzen und Ressourcen zusammenzuführen, für die beteiligten Kliniken nutzbar zu machen und dadurch die Behandlung zu verbessern [16].

Durch die Gründung von Netzwerken wird kostbare Zeit gewonnen, wovon der Patient profitiert. Die Anforderung, Information und Kommunikationswege unter den Kliniken und den Rettungsdiensten sind klar definiert und die Rettungsfahrzeuge entsprechend ausgestattet. Gemeinsame Veranstaltungen wie Workshop, Anwendertreffen und Schulungen bilden die Basis einer guten Zusammenarbeit.

\section{॥) Netzwerke führen benötigte Kompetenzen und Ressourcen zusammen}

Die Optimierung der Zeitabläufe, wie z. B. durch das Anbringen von Aufklebern (- Abb. 6) in Kliniken und HK-Laboren, beispielsweise durch Schulung von internem und externem Personal bezüglich Indikation, Alarmierungsweg sowie Bereitstellung einer 24-h-Bereitschaft und eines Einsatzfahrzeugs durch das ECLSZentrum, ist für den Erfolg des Konzepts ausschlaggebend [17]. 


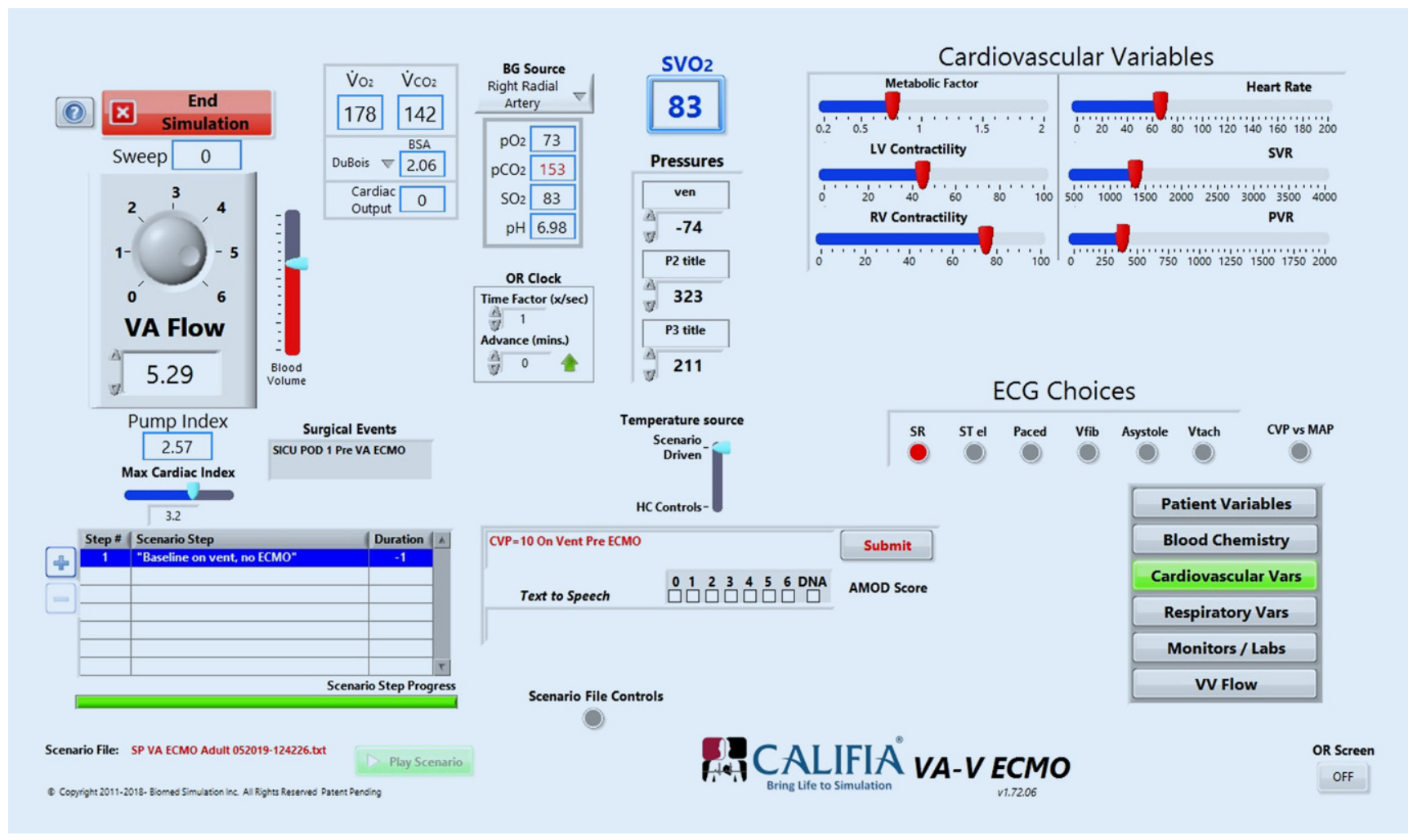

Abb. 7 ॥ Simulator Califa (Mit freundl. Genehmigung $\odot S \& T$ international UG. Alle Rechte vorbehalten.)
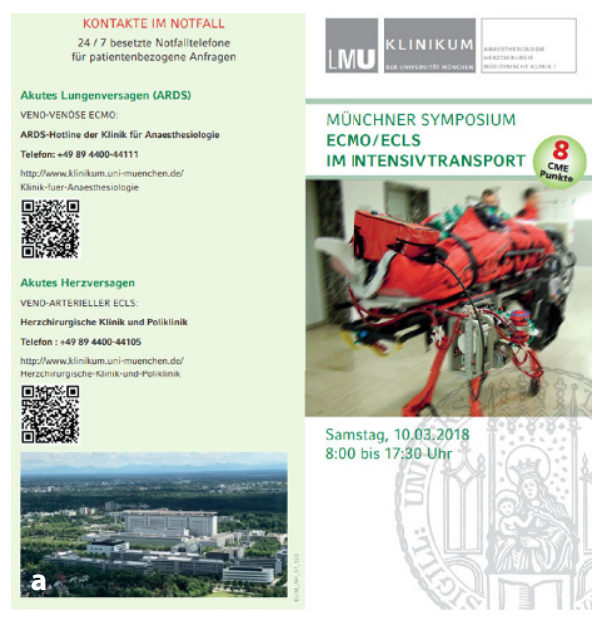

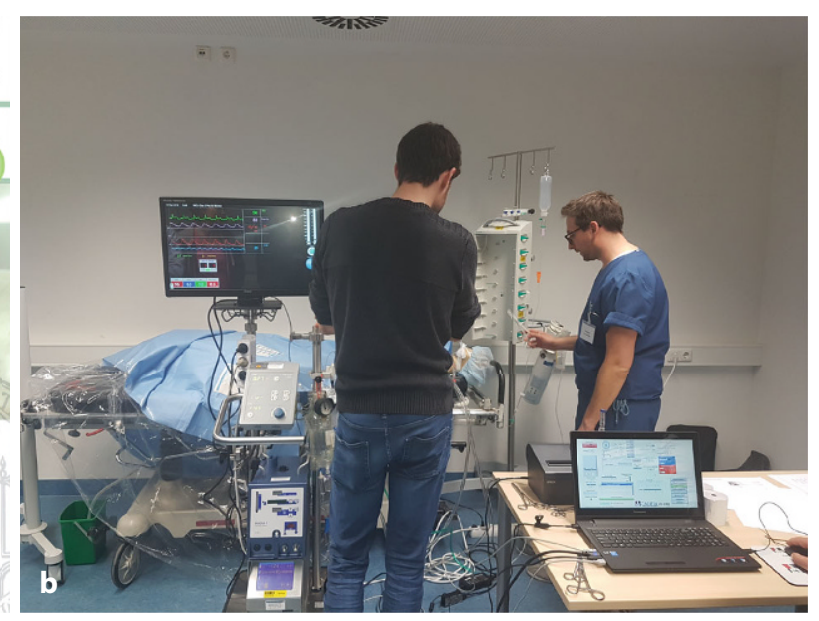

Abb. $8 \triangleleft$ Workshop und Simulation des Extracorporeal Life Support (ECLS) im Zentrum für Simulation an der Ludwig-MaximiliansUniversität ( $L M U)$ München. ECMO extrakorporale Membranoxygenierung. (Mit freundl. Genehmigung $\odot$ LMU, München. Alle Rechte vorbehalten.)

\section{Simulation für den ECMO/ECLS-} Einsatz

Beieiner systematischen Implementierung der ECLS-Therapie ist die Rolle von Training und Simulation nicht allein auf Einzelpersonen beschränkt, sondern ist als "team approach" zu verstehen [18]. An der LMU werden regelmäßige praktische Schulungen und Troubleshooting für alle Berufsgruppen, die Teil der ECLS-Therapie sind, durchgeführt. Dies dient dem Ziel eines ECLS-Zertifikats [19] aufverschiedenen
Berufsebenen. Initiierte ECLS-Zwischenfälle ( $\Delta \mathrm{P}$-Anstieg, Oxygenierungsprobleme etc.) können am Simulator (• Abb. 7) mit allen möglichen Fehlerbehebungsszenarien gezielt geübt werden.

Somit können spezielle Situationen und Notfallszenarien sicher durch realitätsnahes Simulationstraining in einer realistischen Umgebung mit einem ECMO/ECLS-Simulator, echtem medizinischem Equipment und geschulten Instruktoren beherrscht werden, kurz "crew coordination training " für KardiotechnikerIn, Herzchirurgln und Notfallmedizinerln.

\section{Workshop/Symposium}

Rund 70 TeilnehmerInnen aus Kliniken, Intensivpflege, Notarztdienst, Intensivtransport und Rettungsdienst besuchten das 1. ECLS-Symposium in München. Nach einem umfassenden theoretischen Teil über die ECMO-Verfahren wurden die TeilnehmerInnen über die Indikationen der ECLS-Therapie sowie die Besonderhei- 
Tab. 1 Schwerkraftanforderungen an die Haltersysteme bei luftgebundenen Transporten

\begin{tabular}{|l|l|}
\hline Nach oben & $04 \mathrm{G}$ \\
\hline Vorwärts & $16 \mathrm{G}$ \\
\hline Zur Seite & $08 \mathrm{G}$ \\
\hline Nach unten & $20 \mathrm{G}$ \\
\hline Rückwärts & $1,5 \mathrm{G}$ \\
\hline
\end{tabular}

ten beim Transport von Patienten unter ECLS-Therapie, wie sie die DRF Luftrettung im Rahmen von Intensivtransporten durchführt, informiert.

In praxisnahen Workshops (- Abb. 8) konnten in Kleingruppen am Simulator eigene Erfahrungen mit der ECMO/ECLSTherapie gesammelt und die schrittweise Implantation nach der Live-Demonstration einer Kanülierung am Simulator geübt werden.

Im Hangar des ITH Christoph München wurden praktische Aspekte des sicheren Transports dieser kritisch kranken Patienten mit Intensivtransportwagen (ASB Rettungsdienst München) und Hubschrauber erarbeitet. Hierfür wurde durch die DRF Luftrettung eigens ein hochmoderner Hubschrauber vom Typ H145 als Schulungsmaschine bereitgestellt, um unabhängig vom Einsatzgeschehen trainieren zu können. Darüber hinaus findet in den Räumlichkeiten des ASB der Intensivtransportkurs München (gemäß der Deutschen Interdisziplinären Vereinigung für Intensiv- und Notfallmedizin, DIVI) mit dem Schwerpunkt praxisnahe In-situSimulation und ECLS-Transporte statt [20].

\section{\) Diversen Rettungsdiensten wurden die Möglichkeiten der ECLS- Therapie nähergebracht}

Durch regelmäßige Teilnahme des interdisziplinären ECLS-Teams am Münchner Marathon wurde diversen Rettungsdiensten beim Briefing, den Teilnehmern via Pressemitteilungen und Social Media, die Möglichkeiten derECLS-Therapie nähergebracht. Allerdings konnten, bedingt durch die "coronavirus disease 2019" (COVID19), in den Jahren 2020 und 2021 keine Präsenzveranstaltungen durchgeführt werden. Trotzdem konnte ein virtuelles Symposium zum Thema mechanische Herz-Kreislauf-Unterstützung und ECLS

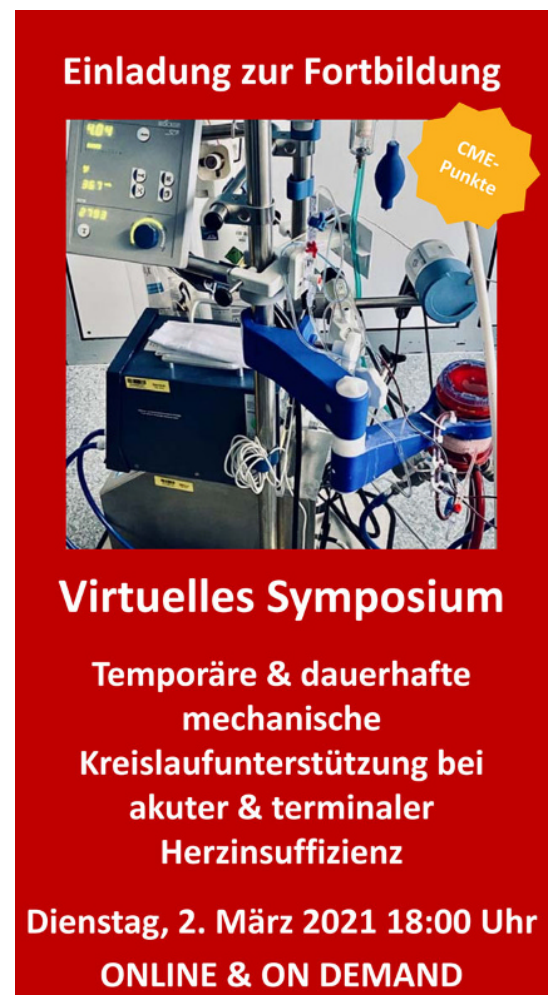

aller Beteiligten ergibt sich eine rasche Verfügbarkeit des ECLS-Teams.

- Die Netzwerkbildung und die Einbindung in die Rettungskette tragen dazu bei, dass das ECLS-Team schnell beim zu versorgenden Patienten eintrifft.

Korrespondenzadresse

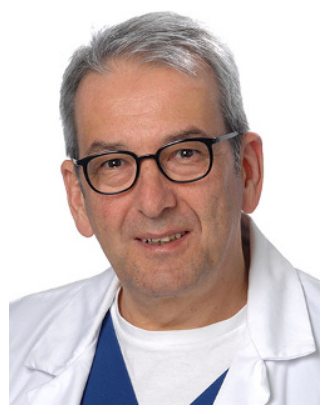

Dr. rer. biol. hum. F. Born

Herzchirurgische Klinik und Poliklinik, Klinikum der LMU

Marchioninistr. 15, 81377 München,

Deutschland

Frank.Born@med.uni-muenchen.de

Abb. $9 \Delta$ Virtuelles Symposium zum Thema mechanische Herz-Kreislauf Unterstützung und Extracorporeal Life Support. (Mit freundl. Genehmigung $\odot$ LMU, München. Alle Rechte vorbehalten)

mit über 200 Teilnehmern stattfinden (• Abb. 9).

\section{Eigene Erfahrungen}

Mehrere Hundert entsprechende Einsätze wurden im Krankenwagen, im Rettungshubschrauber oder im Ambulanzjet durchgeführt [21]. Dabei wurden die Distanzen, die zwischen Krankenhäusern und Spezialzentrum zu überbrücken sind, immer größer. Ein Einsatzradius von 8000 km wurde schon mehrmals deutlich überschritten [22].

\section{Fazit für die Praxis}

- Der Extracorporeal Life Support (ECLS) stellt eine Behandlungschance für einen ausgewählten Teil von Patienten mit therapierefraktärem Herz-Kreislauf-Stillstand dar.

- Eine klare Indikationsstellung ist zwingend, um die komplexe Therapieform sicher und im Sinne des Patienten zum Einsatz zu bringen.

- Durch organisatorische Herausforderungen und ein strukturiertes Teamtraining

\section{Einhaltung ethischer Richtlinien}

Interessenkonflikt. F. Born, C. Müller, M. Hanuna, U. Boeken und C. Hagl geben an, dass kein Interessenkonflikt besteht.

Für diesen Beitrag wurden von den Autoren keine Studien an Menschen oder Tieren durchgeführt. Für die aufgeführten Studien gelten die jeweils dort angegebenen ethischen Richtlinien.

\section{Literatur}

1. Leitlinien zur Reanimation 2015 des European Resuscitation Council, K.G. Monsieurs, J.P. Nolan, L.L. Bossaert et al., Notfall Rettungsmed 2015, 18 : S. $655-747$ 12-05_Zentrums-Regelungen_Erstfassung_ BAnz.pdf. Zugegriffen: 2020

3. Michels G, Wengenmayer T, Hagl C (2019) Empfehlungen zur extrakorporalen kardiopulmonalen Reanimation (eCPR). Z Herz- Thorax- Gefäßchir 33:190-198

4. www.awmf.org/uploads/tx_szleitlinien/ 011-021k_S3_Einsatz-der-extrakorporalenZirkulation-ECLS-ECMO-bei-HerzKreislaufversagen_2021-03.pdf. Zugegriffen: 2021

6. Bisdas T, Beutel G, Warnecke G et al (2011) Vascular complications in patients undergoing femoral cannulation for extracorporeal membrane oxygenation support. Ann Thorac Surg. https:// doi.org/10.1016/j.athoracsur.2011.02.018

7. Berdajs D, Born F, Crosset M et al (2010) Superior venous drainage in the "Life Box": a portable
2. www.g-ba.de/downloads/39-261-4072/2019-

5. www.elso.org.Zugegriffen: 2021 
extracorporeal oxygenator with a self-expanding venous cannula. Perfusion 25:211-215

8. Ouweneel DM, Schotborgh JV, Limpens J,SjauwKD, Engstrom AE, Lagrand WK et al (2016) Extracorporeal life support during cardiac arrest and cardiogenic shock: a systematic review and metaanalysis. Intensive Care Med 42(12):1922-1934

9. Lackermair K, Brunner S, Günther S (2020) Outcome of patients treated with extracorporeal life support in cardiogenic shock complicating acute myocardial infarction: 1-year result from the ECLS-Shockstudy. Clin Res Cardiol. https://doi.org/ 10.1007/s00392-020-01778-8

10. Aubin H, Petrov G, Albert A (2016) A Suprainstitutional network for remote extracorporeal life support A retrospective cohort study. JACC Heart Fail 4(9):698-708

11. Bartos JA, Grunau B, Yannopoulos D (2020) Improved survival with extracorporeal cardiopulmonary resuscitation despite progressive metabolic derangement associated with prolonged resuscitation. Circulation 141:877-886

12. Wang CH, Chen YS, Ma MH (2013) Extracorporeal life support. Curr Opin Crit Care 19:202-207

13. Coppola CP, Tyreeb M, Larryb K, Di Geronimob R (2008) A 22-year experience in global transport extracorporeal membrane oxygenation. J Pediatr Surg 43:46-52

14. Güngerich A (2010) Ein Herz will weiterschlagen. Gönnermagazin Schweizerischen Rettungsflugwacht 74:12-13

15. EASA EASA. www.easa.europa.eu. Zugegriffen: 2020

16. Becker $T$ et al (2011) Netzwerke - praktikabel und zukunftsfähig. In: Becker T (Hrsg) Netzwerkmanagement. Mit Kooperation zum Unternehmenserfolg, 3. Aufl. Springer, Heidelberg

17. Beurtheret S, Mordant P, Paoletti X et al (2013) Emergency circulatory support in refractory cardiogenic shock patientsin remote institutions: a pilotstudy (the cardiac-RESCUE program). Eur Heart J34:112-120

18. Damjanovic D, Gliwitzky B, Deppe M, Benk C, Trummer $G$, Arbeitsgemeinschaft prähospitale eCPR Freiburg (2019) eCPR bei prähospitalem therapierefraktärem Herz-Kreislauf-Stillstand: Praktische Umsetzung im Rettungsdienst und Wissenswertes für Nicht-ECMOlogen. Notfall Rettungsmed 22:124-135

19. Trummer $G$, Müller T, Michels $G$ et al (2021) Ausbildungsmodul Extrakorporaler Life Support (ECLS): Konsensuspapier der DIVI, DGTHG, DGfK, DGAI, DGIIN, DGF, GRC und der DGK. Kardiologe. https://doi.org/10.1007/s00063-021-00815-2

20. www.asb-muenchen.de/unsere-angebote $1 /$ ersten-hilfe-kurse/divi-kurs. Zugegriffen: 2019

21. Güngerich $A$ (2010) Ein Herz will weiterschlagen. Gönnermagazin Schweizerischen Rettungsflugwacht 74:12-13

22. Born F, Ammann U, Burren T, Albrecht Ret al (2010) Transatlantikflug mit transportabler Herz-LungenMaschine "LifeBox". Kardiotechnik (3):65-69. ISSN 0941-26703/2010

\section{Patient transport and networks for use of extracorporeal life support}

New technologies and continuous further development of extracorporeal support systems have expanded the range of applications of extracorporeal life support (ECLS) in recent years. In addition to use in cardiogenic shock or resuscitation, the number of requests for the transfer of unstable patients from peripheral hospitals are increasing. Organizational challenges such as the establishment of networks and structured team training for all parties involved mean that the ECLS team is quickly available to reach the patient.

\section{Keywords}

Cardiopulmonary resuscitation · Organization · Equipment · Complications · Training 